\title{
Ephedra-Induced Gastric Mucosal Injury
}

\author{
Joseph B. Lillegard ${ }^{\mathrm{a}} \quad$ John R. Porterfield Jr. ${ }^{\mathrm{b}}$ \\ aDepartment of Surgery, Mayo Clinic, Rochester, Minn., and ${ }^{b}$ Department of \\ Surgery, University of Alabama Birmingham, Birmingham, Ala., USA
}

\section{Key Words}

Ephedra $\cdot$ Ischemia $\cdot$ Stomach $\cdot$ Mucosa

\begin{abstract}
In this case report we detail the difficult case of a 74-year-old male patient who was transferred to our cardiac intensive care unit in severe shock with an acute abdomen. His abdomen was emergently explored revealing isolated gastric necrosis and ischemia. A subtotal gastrectomy was performed and the patient was discharged from the hospital 34 days after surgery. Postoperatively we learned that for the preceding 2 weeks the patient had doubled the dose of Forcalide syrup which he had been taking for his reactive airway disease for many years. Formal testing of this fluid revealed a concentrated sugar-based solution with a high concentration of both Ephedra and potassium chloride. Final pathology of the resected stomach demonstrated patent vessels to the stomach with extensive microvascular thrombosis and full-thickness gastric necrosis. On final summation of this case we concluded that the Ephedra in the patient's Forcalide syrup caused the microvascular necrosis seen in the stomach and should be added to the list of potential adverse reactions seen with the ingestion of ephedrine.
\end{abstract}

\section{Case Report}

A 74-year-old male presented unconscious to a small community hospital in florid shock. Following prompt intubation and initial hemodynamic stabilization the patient was transferred to our cardiac intensive care unit for a presumed devastating cardiac event. On arrival the patient was found to be in extremis with his initial objective presentation listed in table 1. A history was obtained from the patient's wife who stated that the patient was in his usual state of health until two weeks prior when his reactive airway disease began to worsen with the progression of winter.

For the past 67 years the patient had been taking Karo syrup with ephedrine (Forcalide syrup) every day to treat his reactive airway disease. The patient had carried the diagnosis of reactive airway disease following the accidental ingestion of gasoline at age 7 . Since that time his local pharmacist has prepared the Karol-Ephedrine syrup, which the patient later described had 'always' successfully treated the symptoms of his airway disease. When the patient's symptoms began to worsen two weeks prior to his admission, he doubled his daily intake of the Karol-Ephedrine syrup. During the first week his 
respiratory system improved and the patient was able to return to his usual daily activities. However, during the second week, one week prior to admission, he began experiencing a dull epigastric pain that progressed to periodic bouts of extreme pain. The patient's pain was associated with fever and anorexia, however, he continued to consume the double dose of Karol-Ephedrine syrup. On the morning of presentation the patient's wife found him disoriented, febrile, and experiencing constant extreme abdominal pain.

After initial evaluation by our cardiac critical care colleagues, the diagnosis of septic shock was made with suspicion of an abdominal source. On our initial evaluation he was intubated, sedated but not chemically paralyzed. His abdomen was rigid and markedly distended. His wife reported progression of the severe episodes of epigastric pain. A STAT portable chest and abdominal radiograph demonstrated a massively dilated stomach with decompressed loops of small bowel (fig. 1). A nasogastric tube was placed, which immediately released a rush of air and $200 \mathrm{ml}$ of blood-stained bilious fluid.

The patient was emergently taken to the operating room for exploratory laparotomy. Upon initial abdominal exploration, more than 3 liters of transudative ascites was evacuated which revealed the obvious cause of the patient's sepsis and acute abdomen. The stomach was decompressed due to the placement of a nasograstic tube preoperatively, but showed extensive necrosis extending along the greater curvature and dependent portion of the body of the stomach (fig. 2). Close inspection of the stomach revealed multiple microperforations along the greater curvature where the necrosis was most severe. Interestingly, palpable pulses were present in the vessels supplying the greater curvature of the stomach and there was no evidence of visceral vascular occlusive disease. With the extent of gastric insult a subtotal gastrectomy was required and his intestinal continuity was restored with a Roux-en-Y cardiojejunostomy. The patient tolerated the procedure well and was successfully discharged from the hospital. He discontinued his use of the Karol-Ephedrine syrup and did not have complications associated with gastrointestinal ischemia anymore.

Postoperatively we investigated the cause of the patient's unusual isolated gastric ischemia. Along with a more extensive history, as detailed above, we obtained a sample of the patient's Karol-Ephedrine syrup and tested the liquid along with the patient's serum for the presence of illegal substances and the presence of ephedrine (table 2). The sample of the syrup was submitted for gas chromatography/mass spectrometry (GC/MS) screening. A $2.0 \mathrm{ml}$ sample of the Karol-Ephedrine syrup was combined with $100 \mu \mathrm{l}$ of an internal standard solution $(0.2 \mathrm{mg} / \mathrm{ml}$ benzocaine and $0.2 \mathrm{mg} / \mathrm{ml}$ cyheptamide), acidified with $1 \mathrm{ml}$ buffer ( $\mathrm{pH} 4.0)$ and extracted into $6 \mathrm{ml}$ ethyl acetate/chloroform (20:80 v/v). Extracts were dried under $30^{\circ} \mathrm{C}$ nitrogen and reconstituted in $100 \mu \mathrm{l}$ of pyridine/hexane (10:90). Using an Agilent 5973/6890 GC/MS, $1 \mu \mathrm{l}$ of reconstituted sample was injected onto a DB-5MS column and subjected to gas chromatographic separation. Drugs present were identified by chromatographic retention time compared against a mixture of drug standards, and by structural analysis provided by mass spectrometry [1]. Of the tested substances known to cause vasoconstriction or gastric mucosal injury, ephedrine was the only substance that was found to be present in the patient's Karol-Ephedrine syrup and his serum. Hematoxylin and eosin staining of the gastrectomy specimen demonstrated the presence of chronic gastritis, microvascular thrombosis with full thickness necrosis, and multiple gastric perforations.

\section{Discussion}

Ephedra is an alkaloid chemical compound obtained from the Ephedra sinica plant [2]. In traditional Chinese medicine this plant has been used for millennia to treat disorders such as asthma, upper respiratory illnesses and seasonal allergies [3]. In recent years Ephedra has gained tremendous popularity as a diet drug because of its biochemical activity as a stimulant and a prothermogenic agent $[2,4]$. The stimulant activity of Ephedra suppresses appetite while the prothermogenic property increases the body's metabolism, all leading to improved weight loss [5]. However, over the past decade a number of well-documented reports of ischemic stroke and adverse cardiac events linked to Ephedra have lead to a FDA ban of the drug in April 2004 as a dietary supplement $[2,4,6-12]$.

Ephedra causes a number of physiologic effects in addition to suppressing appetite and increasing metabolism $[2,3,5,12]$. Ephedra causes an increase in blood pressure, heart 
rate and bronchial dilation $[4,7,8,11-13]$. The effect of Ephedra on the vasculature is also well documented and comes from the drugs ability to function as a vasoconstrictor $[8,12,13]$. This effect as a potent vasoconstrictor has lead to the above listed cardiac and cerebral blood flow complications. However, to date no study or case report has shown Ephedra to cause microvascular thrombosis and intestinal necrosis.

The literature contains relatively few examples of isolated gastric ischemia from chemical sources. Ethanol, aspirin and other vasoactive compounds have been implicated in inducing isolated microvascular ischemia in the stomach [14-18]. Ethanol is thought to induce gastric ischemia by stimulating the release of endothelin-1, which is a potent vasoconstrictor [16-18]. Aspirin has been shown to reduce local blood flow, leading to microvascular ischemia and gastric mucosal injury $[14,15,19,20]$.

We conclude that the Ephedra in the patient's Forcalide syrup lead to severe vascoconstriction with resultant microvascular thrombosis and gastric necrosis. Since this case the physicians and the pharmacy have discontinued prescribing or dispensing Forcalide syrup. This is the first case report implicating Ephedra as the cause of gastrointestinal ischemia and gastric perforation. We strongly recommend that this albeit rare scenario be added as a warning to the list of potential adverse reactions seen with the ingestion of Ephedrine.

Table 1. Initial vitals, labs and hemodynamic values at initial presentation to the surgical team

\begin{tabular}{ll}
\hline Initial data at presentation & Values \\
\hline Heart rate, beats/min & 103 \\
Systolic blood pressure, $\mathrm{mm} \mathrm{Hg}$ & 155 \\
Diastolic blood pressure, $\mathrm{mm} \mathrm{Hg}$ & 69 \\
Temperature, ${ }^{\circ} \mathrm{C}$ & 35.1 \\
Central venous pressure, $\mathrm{mm} \mathrm{Hg}$ & 12 \\
Pulmonary capillary wedge pressure, $\mathrm{mm} \mathrm{Hg}$ & 21 \\
Systemic vascular resistance $\left(\right.$ dynes $\bullet$ sec $\left./ \mathrm{cm}^{5}\right)$ & 1,457 \\
Cardiac index, $1 / \mathrm{min} / \mathrm{BSA}$ & 1.83 \\
Hemoglobobin, g/dl & $10.6($ ref. $13.5-17.5)$ \\
Leukocytes $\left(\times 10^{9} / \mathrm{l}\right)$ & $15.4($ ref. $3.5-10.5)$ \\
Platelets $\left(\times 10^{9} / \mathrm{l}\right)$ & 254 (ref. $150-450)$ \\
Lactate, $\mathrm{mmol} / \mathrm{l}$ & 3.9 (ref. $0.6-2.3)$ \\
\hline
\end{tabular}


Table 2. Test results of the patient's Karol-Ephedrine syrup and serum

\begin{tabular}{lll}
\hline $\begin{array}{l}\text { Substances } \\
\text { screened }\end{array}$ & \multicolumn{2}{l}{ Results } \\
\cline { 2 - 3 } & Karol-Ephedrine syrup & serum \\
\hline Caffeine & negative & negative \\
Cocaine & negative & negative \\
Ephedrine & positive & positive \\
Ethanol & negative & negative \\
Ibuprofen & negative & negative \\
Indomethacin & negative & negative \\
Ketoprofen & negative & negative \\
Ketorolac & negative & negative \\
Lidocaine & negative & negative \\
Salicylate & negative & negative \\
\hline
\end{tabular}

Fig. 1. Preoperative abdominal radiograph demonstrated a massively dilated stomach with otherwise normal bowel gas pattern.

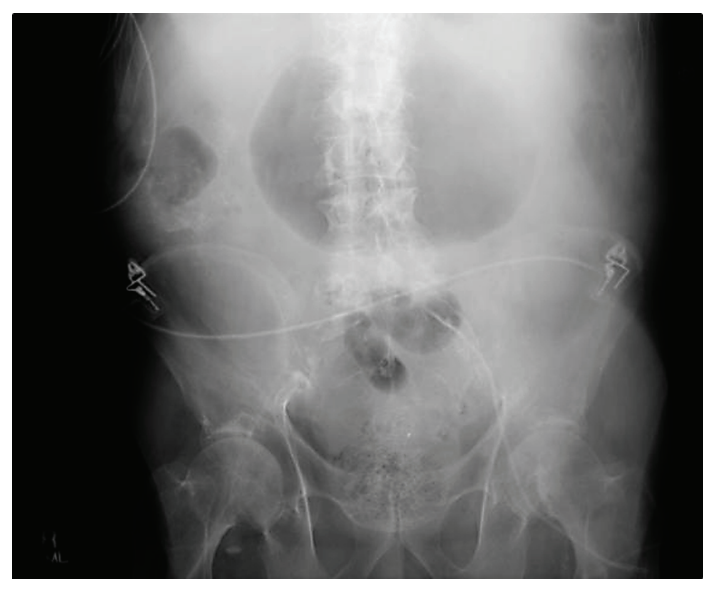

Fig. 2. Intraoperative photograph. The ischemia and necrosis is isolated to the stomach with multiple microperforations noted along the greater curvature.

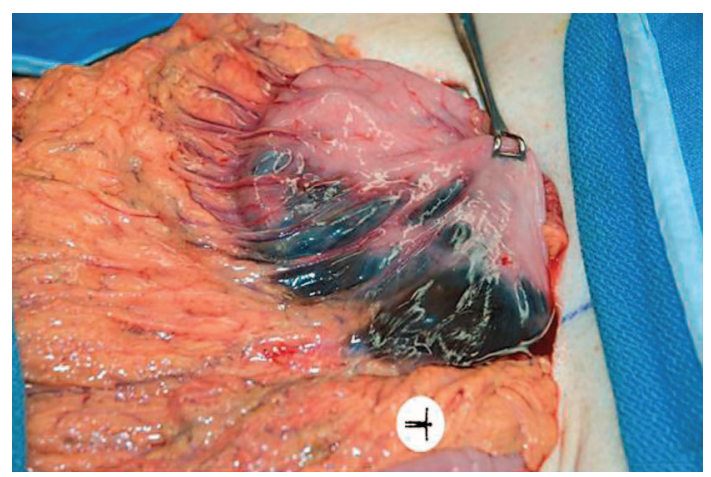




\section{References}

1 Burtis CA, Edward A (eds): Tietz Textbook of Clinical Chemistry, ed 3. Philadelphia, Saunders, 1999, pp 913-917.

2 Gurley BJ, Wang P, Gardner SF: Ephedrine-type alkaloid content of nutritional supplements containing Ephedra sinica (Ma-huang) as determined by high performance liquid chromatography. J Pharm Sci 1998;87:1547-1553.

3 Abourashed EA, El-Alfy AT, Khan IA, Walker L: Ephedra in perspective - a current review. Phytother Res 2003;17:703-712.

4 Haller CA, Benowitz NL: Adverse cardiovascular and central nervous system events associated with dietary supplements containing ephedra alkaloids. N Engl J Med 2000;343:1833-1838.

5 Bent S, Tiedt TN, Odden MC, Shlipak MG: The relative safety of ephedra compared with other herbal products. Ann Intern Med 2003;138:468-471.

6 Blechman KM, Karch SB, Stephens BG: Demographic, pathologic, and toxicological profiles of 127 decedents testing positive for ephedrine alkaloids. Forensic Sci Int 2004;139:61-69.

7 Bouchard NC, Howland MA, Greller HA, Hoffman RS, Nelson LS: Ischemic stroke associated with use of an ephedra-free dietary supplement containing synephrine. Mayo Clin Proc 2005;80:541-545.

8 Chen C, Biller J, Willing SJ, Lopez AM: Ischemic stroke after using over the counter products containing ephedra. J Neurol Sci 2004;217:55-60.

9 Fleming RM: The effect of ephedra and high fat dieting: a cause for concern! A case report. Angiology 2007;58:102-105.

10 Munarriz R, Hwang J, Goldstein I, Traish AM, Kim NN: Cocaine and ephedrine-induced priapism: case reports and investigation of potential adrenergic mechanisms. Urology 2003;62:187-192.

11 Rezkalla SH, Mesa J, Sharma P, Kloner RA: Myocardial infarction temporally related to ephedra - a possible role for the coronary microcirculation. WMJ 2002;101:64-66.

12 Samenuk D, Link MS, Homoud MK, Contreras R, Theoharides TC, Wang PJ Estes NA: Adverse cardiovascular events temporally associated with ma huang, an herbal source of ephedrine. Mayo Clin Proc 2002;77:12-16.

13 Nyska A, Murphy E, Foley JF, Collins BJ, Petranka J, Howden R, Hanlon P, Dunnick JK: Acute hemorrhagic myocardial necrosis and sudden death of rats exposed to a combination of ephedrine and caffeine. Toxicol Sci 2005;83:388-396.

14 Ashley SW, Sonnenschein LA, Cheung LY: Focal gastric mucosal blood flow at the site of aspirin-induced ulceration. Am J Surg 1985;149:53-59.

15 Binmoeller KF, Benner KG: Emphysematous gastritis secondary to gastric infarction. Am J Gastroenterol 1992;87:526-529.

16 Kawano S, Masuda E, Tsuji S, Nagano K, Fusamoto H, Kamada T: Ethanol causes vasoconstriction due to endothelin-1 release in rabbit gastric vessels. Microvasc Res 1991;41:408-410.

17 Masuda E, Kawano S, Nagano K, et al: Effect of ethanol on endothelin-1 release from gastric vasculature. Gastroenterol Jpn 1991;26(suppl 3):81-82.

18 Masuda E, Kawano S, Nagano K, Tsuji S, Ishigami Y, Tsujii M, Hayashi N, Fusamoto H, Kamada T: Effect of intravascular ethanol on modulation of gastric mucosal integrity: possible role of endothelin-1. Am J Physiol 1992;262:G785G790.

19 Pettei MJ, Levy J, Abramson S: Nonocclusive mesenteric ischemia associated with propranolol overdose: implications regarding splanchnic circulation. J Pediatr Gastroenterol Nutr 1990;10:544-547.

20 Pfeiffer CJ, Keith JC, Cho CH, DeRolf S, Pfeiffer DC, Misra HP: Gastric and cardiac organoprotection by lidocaine. Acta Physiol Hung 1989;73:129-136. 